\title{
Detecting Tropical Forest Biomass Dynamics from Repeated Lidar
}

\section{Measurements}

\section{Supplementary Material}

\section{(1) Lidar Data Acquisition}

LVIS (Laser Vegetation Imaging Sensor) is a medium altitude waveform digitizing Lidar

measuring canopy height, ground elevation, and the waveform representing the vertical profile or the distribution of intercepted surfaces within the Lidar footprint (Hofton et al., 2002). Its expanded spatial coverage allows large scale mapping of topography, forest structure, and AGB (Weishampel et al., 2000; Drake et al., 2002a; Drake et al., 2002b). LVIS Lidar data were collected by NASA over BCI in 1998 (Blair et al., 1999; Dubayah and Drake, 2000). This 1998 Panama LVIS survey consists of 215,984 individual LVIS shots, of which 98,040 are located over BCI and used for this study. LVIS's large footprint ( $20 \mathrm{~m})$ generally exceeds the average crown diameter of large trees (King, 1996; Drake et al., 2003).

The airborne discrete-return Lidar (DRL) that acquired data in 2009 was a small footprint instrument, ranging between $0.25 \mathrm{~m}$ and $0.60 \mathrm{~m}$ footprint. It is a proven technique in quantifying sub-canopy topography and providing accurate vertical forest structure (Dubayah and Drake, 2000; Hyyppa et al., 2001; Lefsky et al., 2002). The DRL data was collected at very low altitude (between 500 and 1500m) and included repeated passes, resulting in multiple measurements per square meter (up to 10 points or echoes per square meter) for precise characterization of vegetation structure.

DRL data were collected by Blom Corporation and Northrop Grumman as part of an NSF funded project, using an Optech 3100 Lidar scanning at a rate of $70 \mathrm{Khz}$. The data were 
collected over 11 individual flights yielding a total of over 233 million laser shots, and over 528 million individual points, resulting in an average point density of 5.6 points per square meter $\left(\mathrm{ppm}^{2}\right)$, and 8.1 returns per square meter $\left(\mathrm{rpm}^{2}\right)$. The DRL data was postprocessed by BLOM Corporation using Bentley's MicroStation to calibrate and filter the data. In addition to the automated filtering process, additional manual editing of the subcanopy DEM was performed to produce a bare-earth DEM product. To ensure accuracy and compliance with the precision requirements, the ground surface was tested using 36 ground surveyed points on flat, hard, well defined surfaces, free of obstacles. The results of the vertical accuracy assessment determined an average error in height of $-0.069 \mathrm{~m}$, an RMSE value of $0.076 \mathrm{~m}$ and a standard deviation of $0.032 \mathrm{~m}$.

\section{(2) LVIS Calibration}

To compare LVIS and DRL Lidar data for changes in vegetation structure requires the data to be cross-calibrated. We performed the cross-calibration of the sensors such that both provide the same ground elevation under forest canopy (Fricker et al., 2012). LVIS, being a large footprint Lidar (20-25 m) has a limited ability to detect ground in dense canopy forests, particularly in complex topographies, thus affecting vegetation height metrics and potentially causing errors in AGB estimations (Dubayah et al., 2010). In contrast, the DRL sensor provides an accurate estimate of ground elevation and vegetation height. A shortened LVIS laser profile will result in a general underestimation of biomass and vertical stratification.

We found that the average vertical difference between the 98,040 LVIS last-return points and the DRL ground surface across BCI was of $28.7 \mathrm{~cm}$. Although the majority $(82.3 \%)$ 
of all LVIS points matched discrete return elevations to 2 meters or less, significant LVIS last-return outliers were identified, ranging from $16.4 \mathrm{~m}$ below the ground surface, to last-return points over $35.7 \mathrm{~m}$ above the ground surface across the island. Also, areas of high terrain slope show consistently more error in large-footprint Lidar ground detection. Because our goal is to obtain optimal AGB estimations and to reduce errors due to the sensors, LVIS data had to be corrected before it was used in our AGB estimation algorithm.

LVIS data was corrected using a method developed by Fricker et al (2012). It uses slope to correct LVIS semi-automatically. They use the LVIS sub-canopy Digital Elevation Model (DEM) alone to estimate slope and apply the effects of terrain slope on subcanopy topography. However, for the present study we had high-resolution Lidar data (DRL) over the whole island, so we simply used the DRL DEM as a reference for subcanopy topography. The elevation difference between DRL and LVIS subcanopy topography was calculated for each LVIS shot by comparing the LVIS ground elevation to the DRL elevation. This difference (positive or negative) was then added to each LVIS vertical height metrics. The result is a more accurate LVIS digital elevation model and improved LVIS estimates of forest structure (Fig. S1). Note that it is difficult to quantify the improvements made by these corrections at the plot level on AGB estimations because there are only a few outliers in this part of the island. 



Elevation

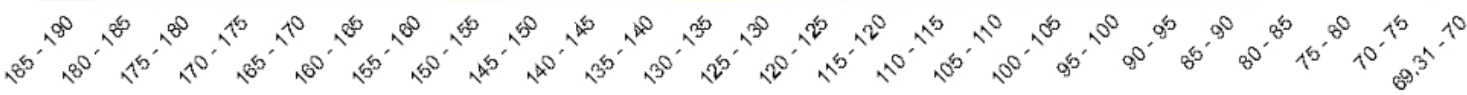

Figure S1 : LVIS ground correction. Raw LVIS ground returns (left) and corrected LVIS ground returns (right) using the DRL Digital Elevation Model.

In spite of the above corrections, DRL and LVIS present too many differences to be used the same way to estimate AGB. They do not have the same footprint size, which makes it impossible to do a footprint to footprint comparison as done in Dubayah et al. (2010).

Furthermore, LVIS height metrics were directly calculated for each shot during postprocessing, whereas we calculated the DRL height metrics using the vertical histogram of canopy height for a given pixel size (at 20m, 50m and 100m resolutions). For these reasons, intermediate height metrics are different for DRL and LVIS. Consequently, we used DRL and LVIS metrics independently in the determination of regression models for AGB estimations. 


\section{(3) Lidar Height Metrics}

To develop the relative height $(\mathrm{RH})$ metrics at each scale, LVIS and DRL data were extracted using shape files partitioning the 50ha plot into respectively, 1250, 200 and 50 regions of interest representing the spatial scales of the analysis.

From LVIS waveform data, relative height quartiles RH25, RH50, RH75 and RH100 were produced for each Lidar shot, where the RH100 metric represents the canopy top height or the maximum height of trees within the Lidar footprint. We aggregated all the shots whose center coordinates fell into a given subplot to calculate the average of the LVIS RH metrics, rather than having their whole footprint contained in the plot, as done previously (Dubayah et al., 2010). This choice was based on the sizes of the ground subplots used in this study. Because LVIS footprint is approximately $20 \mathrm{~m}$, very few shots fall entirely in a $20 \mathrm{~m} * 20 \mathrm{~m}$ subplot. In addition to RH100, which is the average of all the LVIS RH100 within a subplot, we also used RH100max, as the maximum of RH100s of LVIS shots falling in each subplot to get the "real" RH100 comparable to DRL RH100 for each subplot.

A similar approach was used to convert the canopy height data from DRL at $1 \mathrm{~m}$ resolution to waveforms at the same spatial scales. The relative height metrics (RH25, RH50, RH75 and RH100) were produced from these waveforms. The least important height metric in explaining the overall variations of biomass is $\mathrm{RH} 25$, representing the height of the lowest part of the canopy to the forest floor. Although the RH metrics are individually correlated to the ground-based AGB, using the four of them improves the $\mathrm{R}^{2}$ and the RMSE of the ground-based AGB vs. Lidar-based AGB (Fig. S2). Using the metrics RH25, RH50, RH75 and RH100 at the 1 ha scale $\left(\mathrm{R}^{2}=0.71\right.$ and $\mathrm{RMSE}=26.0$ 
for LVIS, $\mathrm{R}^{2}=0.75$ and $\mathrm{RMSE}=24.8$ for DRL, gives more accurate results than when RH25 is left out of the models $\left(\mathrm{R}^{2}=0.72\right.$ and $\mathrm{RMSE}=26.2$ for $\mathrm{DRL}$ and $\mathrm{R}^{2}=0.75$ and RMSE $=26.1$ for LVIS).
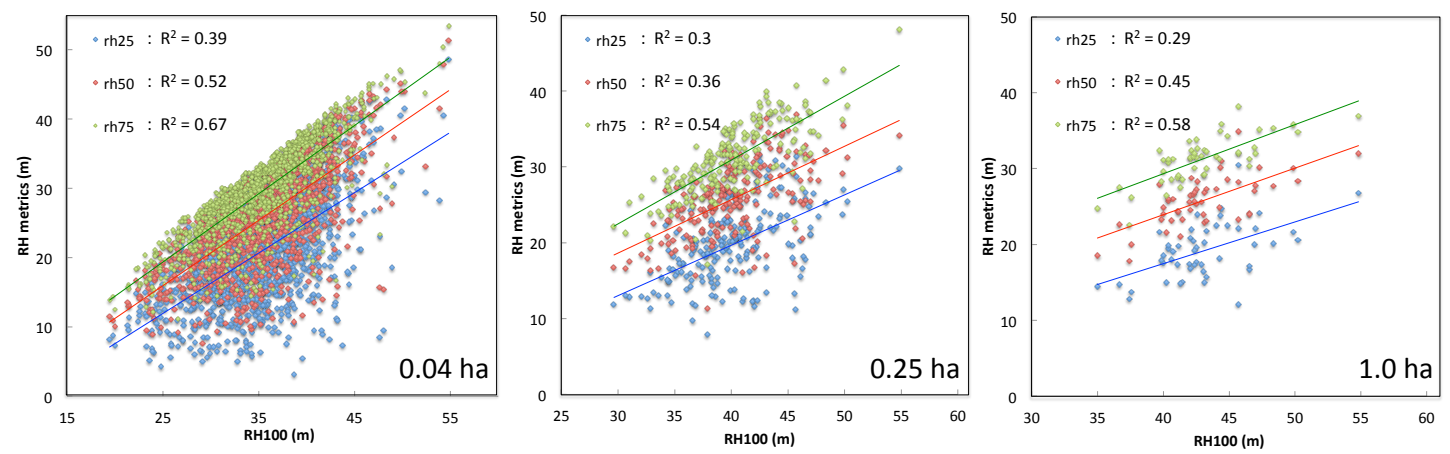

Figure S2: The relationship between the DRL height metrics and the maximum height of the canopy derived at three spatial scales of $0.04,0.25$, and 1.0 ha (left to right). In all cases, the correlations among the height metrics are strong but with significant variations that allow all four metrics contribute to the biomass estimation.

The analysis for relative contribution for height metrics and the stepwise analysis using the Akaike's information criteria (AIC) suggest that the inclusion of RH25 at 1-ha spatial scale contributes similar to RH100 to explain the biomass variations. The relative contribution stays almost the same across scales with the four height metrics explaining about $75 \%$ of variations at 1 -ha, $58 \%$ at 0.25 ha and about $32 \%$ at the 0.04 ha. Using data from a much wider range of biomass such as low density secondary forests, may improve the correlation of one or two height metrics (RH50 and RH75) with biomass and reduce the effect of other metrics (RH100, RH25). However, concentrating on old growth forest alone, all four metrics contribute significantly to estimate forest biomass. 


\section{(4) Spatial Scale}

Fig. S3 illustrates why using small plots for estimating AGB in tropical forests may result in an improper AGB estimation. Because tree crowns can reach over $20 \mathrm{~m}$ in diameter, chances are that a tree crown will significantly overlap several adjacent $20 \mathrm{~m} * 20 \mathrm{~m}$ subplots, thus contributing to the Lidar signal of more than one subplot. This yields to serious problems when attempting to correlate the Lidar signal and ground-based AGB estimations because the ground measurement of a tree (i.e., the physical location of the stem) only contributes to the AGB of a single subplot. This border effect declines as the subplot size increases to $50 \mathrm{~m} * 50 \mathrm{~m}$ and $100 \mathrm{~m} * 100 \mathrm{~m}$ subplots, although edge effects are still expected to be present. At 1ha, the contribution to canopy heights from trees rooted outside the plot boundary becomes small compared to those that do not transgress the plot boundaries.



(a)

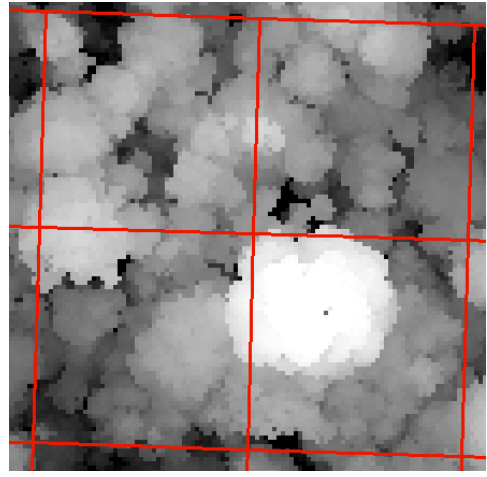

(b)

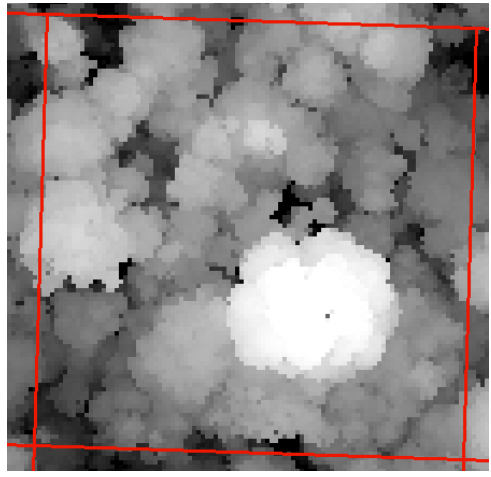

(c)

Figure S3 : Effects of plot size on DRL canopy height share. At 0.04 ha (a), a big tree is predominant in two subplots and present in two others. At $0.25 \mathrm{ha}(\mathrm{b})$, the big tree is mainly present in one subplot, but other trees are split between subplots. At 1ha (c), the edge effect is still there but it becomes negligible compared to the number of trees that are fully contained in the subplot. 


\section{(5) AGB estimation from ground measurements}

Above-ground biomass density was estimated using an allometric regression model for moist tropical forests (Chave et al., 2005). The allometric method is based on the argument that the total aboveground biomass (AGB, in $\mathrm{kg}$ ) of a tree with diameter D, measured at the breast height $(\mathrm{DBH}) 1.3 \mathrm{~m}$ above ground must be proportional to the product of wood specific gravity $(\rho)$ and tree stem volume. Stem volume, in turn, is proportional to the product of stem basal area and total tree height $(\mathrm{H})$ :

$$
A G B=\exp \left[-2.977+\ln \left(\rho D^{2} H\right)\right] \cong 0.0509 \times \rho D^{2} H
$$

Where $\cong$ represents the mathematical identity meaning both formulas can be used in biomass estimation procedure. In the above equation, $\mathrm{D}$ (in $\mathrm{cm}$ ) is measured during the inventory census periods, $\rho$ (in $\left.\mathrm{g} / \mathrm{cm}^{\wedge} 2\right)$ is provided from an available table of measurements for 123 species available in BCI, and tree height $\mathrm{H}$ (in $\mathrm{m}$ ). Tree height measurements were not available for all the trees in the 50ha plot, so we developed a relationship between DBH and height of 1835 trees among them 1604 trees with DBH> $10 \mathrm{~cm}$, representing 154 species (Fig S4). The equation is provided in the log form for the entire range after testing different fits to the data with difference DBH range:

$$
H=-11.731+22.766 \log (D), D>10 \mathrm{~cm}
$$

We then used the height estimations as estimated from equation (ES2) into equation (ES1) for AGB estimation. 


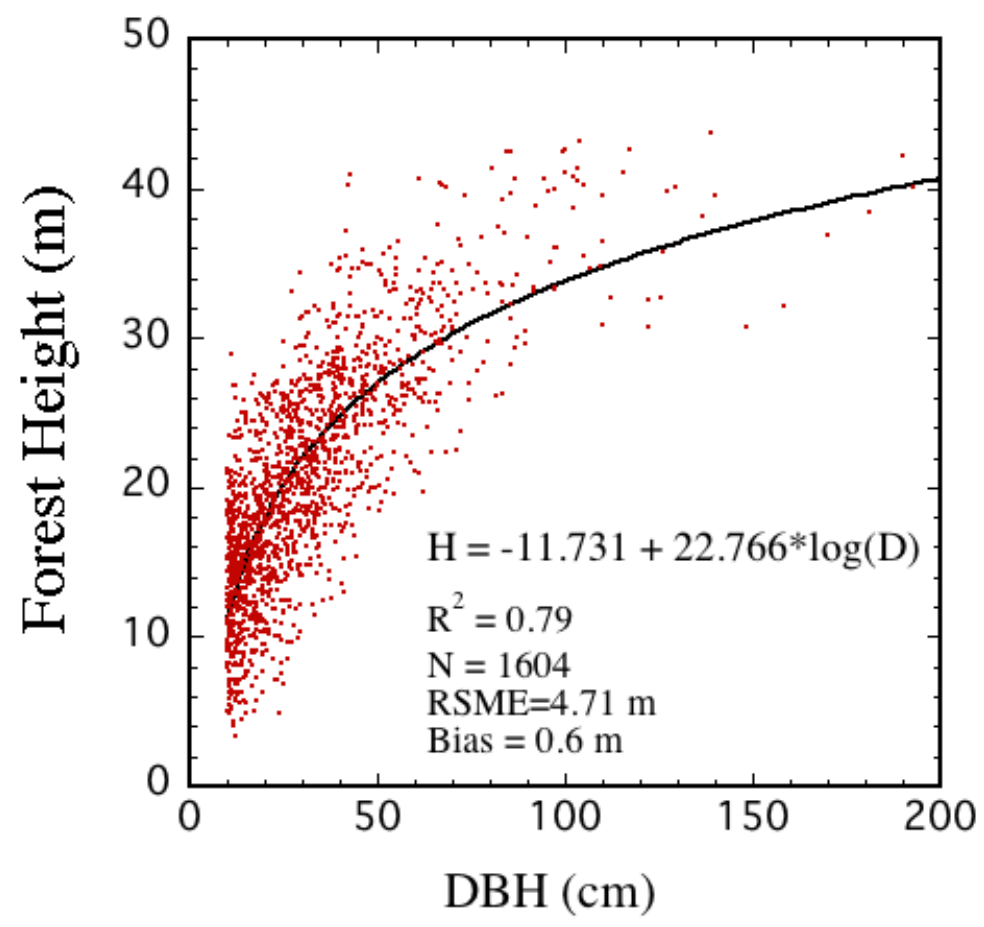

Figure S4 : Relationship between DBH (cm) and tree height $\mathrm{H}$ (in $\mathrm{m}$ ).

The census data was filtered for anomalous and erroneous DBH measurements by first identifying all trees with growth larger than $45 \mathrm{~mm} / \mathrm{year}$ and less than $-5 \mathrm{~mm} / \mathrm{yr}$. The $\mathrm{DBH}$ of these trees were replaced using growth rates equal to mean growth rate of trees in the same DBH class (Condit et al. 1993, 1999). The approach was extended to 2000, 2005, and 2010 when estimating biomass and biomass change from ground data. We calculated the errors associated with biomass estimation for each census at the scale of analysis and used these errors when analyzing the Lidar estimation of biomass and biomass change. See Chave et al., (2003) for more details on census data trimming procedures. 


\section{References}

Blair, J.B., Rabine, D.L., Hofton, M.A., 1999. The Laser Vegetation Imaging Sensor: a medium-altitude, digitisation-only, airborne laser altimeter for mapping vegetation and topography. ISPRS Journal of Photogrammetry and Remote Sensing 54, 115-122.

Chave, J., Condit, R., Lao, S., Caspersen, J.P., Foster, R.B., Hubbell, S.P., 2003. Spatial and temporal variation of biomass in a tropical forest: results from a large census plot in Panama. Journal of Ecology 91, 240-252.

Condit, R., Ashton, P.S., Manokaran, N., LaFrankie, J.V., Hubbell, S.P., Foster, R.B., 1999. Dynamics of the forest communities at Pasoh and Barro Colorado: comparing two 50, Äiha plots. Philosophical Transactions of the Royal Society of London. Series B: Biological Sciences 354, 1739-1748.

Condit, R., Hubbell, S.P., Foster, R.B., 1993. Identifying fast-growing native trees from the neotropics using data from a large, permanent census plot. Forest Ecology and Management 62, 123-143.

Dubayah, R.O., Drake, J.B., 2000. Lidar Remote Sensing for Forestry. Journal of Forestry 98, 44-46.

Hofton, M.A., Rocchio, L.E., Blair, J.B., Dubayah, R., 2002. Validation of Vegetation Canopy Lidar sub-canopy topography measurements for a dense tropical forest. Journal of Geodynamics 34, 491-502.

Hyyppa, J., Kelle, O., Lehikoinen, M., Inkinen, M., 2001. A segmentation-based method to retrieve stem volume estimates from 3-D tree height models produced by laser scanners. Geoscience and Remote Sensing, IEEE Transactions on 39, 969-975.

King, D.A., 1996. Allometry and Life History of Tropical Trees. Journal of Tropical Ecology 12, 25-44.

Weishampel, J.F., Blair, J.B., Knox, R.G., Dubayah, R., Clark, D.B., 2000. Volumetric lidar return patterns from an old-growth tropical rainforest canopy. International Journal of Remote Sensing 21, 409 - 415. 